\title{
Intertextualidad y estrategias traductoras. Sobre algunas versiones de un pasaje de En la orilla (Rafael Chirbes, 2013)
}

\author{
EUGENIO MaGgI \\ Universidad de Bolonia
}

Título: Intertextualidad y estrategias traductoras. Sobre algunas versiones de un pasaje de En la orilla (Rafael Chirbes, 2013).

Resumen: Esta breve nota avanza algunas observaciones sobre cómo las recientes traducciones alemana, italiana, francesa e inglesa de la novela de Rafael Chirbes En la orilla (2013) han abordado un pasaje especialmente rico en referencias intertextuales (la Biblia, Villon, Manrique).

Palabras clave: Rafael Chirbes, En la orilla, intertextualidad, traducción, Jorge Manrique.

Fecha de recepción: 20/11/2015.

Fecha de aceptación: 28/11/2015.
Title: Intertextuality and Translation Strategies. On some Versions of a Passage from Rafael Chirbes' En la orilla (2013).

Abstract: This short note makes some observations on how the recent translations into German, Italian, French and English of Rafael Chirbes' novel En la orilla (2013) have faced a passage especially rich in intertextual allusions (the Bible, Villon, Manrique).

Key words: Rafael Chirbes, En la orilla, Intertextuality, Translation, Jorge Manrique.

Date of Receipt: 20/11/2015.

Date of Approval: 28/11/2015.

Disertando desde las páginas de El lector desprevenido sobre la potencial opacidad de las alusiones intertextuales para el público contemporáneo, Ricardo Senabre comentaba, entre varios ejemplos, dos pasajes de la última novela de Rafael Chirbes, En la orilla (2013). El segundo de ellos, que forma parte de la brevísima sección tercera de la obra, titulada Éxo$d o$, resulta especialmente sugestivo, pues contiene, en palabras del propio Senabre, "una brillante reescritura -que es a la vez una sorprendente 
actualización- de algunas de las Coplas de Jorge Manrique", con "ecos de Bécquer, Villon y Góngora”. De esta larga secuencia, selecciono los fragmentos más relevantes para los propósitos de este artículo:

[...] es hora de llanto y crujir de dientes, de arrepentimientos: ‘adónde fueron los euros de antańo?, ¿qué se hizo de aquellos hermosos billetes morados? Cayeron deprisa como hojas muertas de otoño en día de ventolera, y se pudrieron en el barro: cayeron sobre las mesas de juego, entre las pinzas de los bogavantes y bueyes de mar que hacíamos crujir con las tenazas de descascarillar marisco [...], en los camastros de los prostíbulos; sobre las cisternas de los retretes en los que se espolvoreó polvo blanco [...]: los hermosos billetes morados de quinientos, $u b i$ ? ¿ ¿dónde han ido a parar? Los busca todo el mundo y nadie los encuentra, los buscamos los empresarios, los buscan los funcionarios de hacienda, nada por aquí, nada por allá. [...] Todo se fue por los desagües, por los fregaderos, por los retretes, por el agujero de los cońos apenas en flor y ya encallecidos de tanto frotar. La vida misma, la nuestra, tampoco creas que se va por otro sitio, el mundo entero se vierte por el desagüe, pero cómo echamos de menos todas esas cosas que no volverán. Las nieves de antańo, las rosas que se han abierto esta mañana y se marchitarán a la tarde y cuando vuelva a darles el sol se quedarán sin pétalos, feas bolas secas, pequeñas calaveras que crujen entre los dedos cuando las aplastas, los infantes de Olba, las damas de Ucrania. Adónde fue toda esa gente que pasaba deprisa ante nuestros ojos, adónde iban, en qué pararon. Agua que se traga el fregadero, laberinto de cañerías, cloacas, filtros y balsas depuradoras, tubos que van a dar a la mar².

Este cínico monólogo interior del contratista estafador Tomás Pedrós (quien, ante un epocal derrumbe de economías y existencias, acaba recomendándole a su mujer Amparo que siga acumulando dinero convertible, joyas y lingotes de oro) delata de forma emblemática el recelo que le inspiraba a Chirbes el poder hechicero de la cultura, ese "animal que anda por todas partes, de naturaleza enredadora, y sobre cuyas intencio-

1 Ricardo Senabre, El lector desprevenido, Oviedo, Ediciones Nobel, 2015, pp. 325326 (versión ebook).

2 Rafael Chirbes, En la orilla, Barcelona, Anagrama, 2013, pp. 433-435. 
nes dominadoras conviene no crearse ninguna esperanza" ${ }^{3}$. Desde luego, las palabras de Pedrós son perfectamente complementarias con el anterior desahogo del carpintero Esteban, que con su terminal rencor de clase desmantela la hipocresía ideológica del contemptus mundi, aquí plasmada por los discursos de Francisco, sempiterno vencedor:

No es verdad lo que te dicen, eso de que viniste sin nada y te irás sin nada. [...] [N] o puedes decirme que viene a ser lo mismo, vanidades y nada más que vanidades, que era lo que decías cuando aún eras cristiano, nerviosa larva de cura obrero ${ }^{4}$.

Cabría ahondar en la fuerza retórica con la que Chirbes ofició el réquiem por una civilización infame, registrando los últimos estertores de la picaresca corte de un pueblo imaginario (los infantes de Olba) y de sus prostibularias damas de Ucrania, y observar cómo pocos meses después otra preciosa mirada crítica, la de Isaac Rosa, vapuleaba de manera similar la pasividad cómplice de su propia generación, la nacida en los 70, con su "tópico recuento de las vidas que ya no viviríamos, las decisiones irreversibles, las oscuras golondrinas y todo ese ubi sunt que sonaba a estribillo pop" ${ }^{\prime}$.

En esta ocasión, sin embargo, reanudando desde una perspectiva traductora las reflexiones que avanzaba Senabre sobre las implicaciones comunicativas de la intertextualidad, me interesa analizar cómo un pasaje tan denso como el que he citado al comienzo del artículo puede conservar o perder su potencial al trasponerse a diferentes idiomas y contextos culturales. El corpus de traducciones que utilizaré, organizado en orden cronológico, es el siguiente ${ }^{6}$ :

3 Juan Manuel Ruiz Casado, "El fracaso de la cultura en las novelas de Rafael Chirbes”, Turia, 112 (2014), pp. 201-207 (p. 203).

4 Ibidem, pp. 361-362.

5 Isaac Rosa, La habitación oscura [2013], Barcelona, Seix Barral/Booket, 2015, p. 74.

6 Cf. los datos bibliográficos básicos aportados por Fernando del Val, "Biocronología de Rafael Chirbes", Turia, 112 (2014), pp. 280-305, actualizados hasta octubre de 2014. En esta sede, por falta de competencias lingüísticas, no consideraré la traducción al neerlandés (Aan de oever), al cuidado de Eugenie Scholderman y Arie van der Wal, que publicó la editorial Meridiaan en octubre de 2014. 
- Am Ufer, traducción de Dagmar Ploetz, München, Antje Kunstmann, enero de 2014.

- Sulla sponda, traducción de Pino Cacucci, Milano, Feltrinelli, septiembre de 2014.

- Sur le rivage, traducción de Denise Laroutis, Paris, Payot \& Rivages, enero de 2015.

- On the Edge, traducción de Margaret Jull Costa, New York, New Directions, enero de 2016 .

En cuanto a los idiomas francés y alemán, nos encontramos con dos traductoras históricas de Chirbes: Laroutis fue vertiendo con regularidad las novelas del autor valenciano para Payot \& Rivages desde finales de los 90 (Tableau de chasse, 1998), mientras que Dagmar Ploetz es su traductora oficial al alemán a partir del año 2000, cuando Kunstmann, editor de Chirbes desde 1996, publicó Der Fall von Madrid. No sobra observar que estas sólidas relaciones han acompańado al reconocimiento de Chirbes en los respectivos ámbitos lingüísticos y culturales, con un éxito de crítica y público que antecede varios años el caso nacional de Crematorio $(2007)^{8}$.

La traducción al italiano, publicada en 2014 por la importante editorial Feltrinelli, fue firmada por Pino Cacucci, conocido novelista y traductor (es suya, entre otras muchas, la versión italiana de Soldados de Salamina de Cercas), con el cual Chirbes presentó la obra en el Festival della Letteratura de Mantua?.

Solo falta mencionar la traducción más reciente, al cuidado de la experta Margaret Jull Costa, que a lo largo de su notable carrera ha vertido al inglés numerosas novelas de Marías, Atxaga, Saramago y Eça de Queirós.

7 Agradezco a New Directions el envío de una copia promocional de la novela antes de su publicación oficial, prevista para finales de enero de 2016.

8 Sobre el caso alemán, especialmente significativo, pueden leerse Javier Aniorte, "Rafael Chirbes, traducción y reconocimiento en Alemania: Los paisajes del alma”, en Las letras valencianas en la literatura universal. Problemas de recepción y traducción: el paisaje y el tiempo, eds. Juan Antonio Albaladejo Martínez y Miguel Ángel Vega Cernuda, Sevilla, Bienza, 2012, pp. 149-156; Jorge Herralde, "Informe sobre una apoteosis a cámara lenta: Rafael Chirbes", Turia, 112 (2014), pp. 176-190; José Manuel López de Abiada, "Un sabueso a la caza de la verdad. Calas en la recepción crítica de Rafael Chirbes en Alemania”, Turia, 112 (2014), pp. 191-200.

9 Puede verse un vídeo del encuentro en https://www.youtube.com/watch?v=Iny9b-GvY4Q. 
Es importante añadir que Laroutis y Cacucci utilizan un aparato de notas, bastante esencial y enfocado sobre todo en datos históricos y realia, mientras que Ploetz y, en particular, Jull Costa suelen adoptar otras estrategias (glosas intratextuales, adaptaciones, ocasionales omisiones) para colmar la falta completa de comentarios paratextuales. En ninguna de las traducciones consideradas, de todas formas, hay notas o aclaraciones explícitas sobre el pasaje que he citado arriba, cuyas traducciones paso ahora a copiar para facilitar su posterior análisis:

[...] es ist die Stunde des Heulens und Zähneklapperns, der Reue: Wo sind sie hin, die Euros von einst, was ist aus diesen wunderhübschen zartvioletten Scheinen geworden, sie sind schnell gefallen, wie tote Blätter im herbstlichen Wind, und im Matsch verfault, sie sind auf die Spieltische gefallen, auf die Betten der Bordelle, zwischen die Scheren der Hummer und Taschenkrebse, die wir mit den Zangen für Schalentiere knackten [...]; aufgegangen in weißem Pulver, gestäubt auf die Wasserspeicher der Toiletten [...]; die wunderschönen zartvioletten Fünfhunderterscheine, ubi sunt? Wo sind sie hin? Alle Welt sucht sie, und niemand findet sie, wir Unternehmer suchen sie, die Finanzbeamten suchen sie, nichts hier, nichts dort. [...] Alles ist durch die Abflüsse, durch die Spülbecken, die Toiletten, durch das Loch der noch kaum erblühten, aber vom vielen Reiben schon mit Hornhaut behafteten Mösen abgegangen. Das Leben selbst, unser Leben, glaub ja nicht, dass das woandershin fließt, die ganze Welt fließt mit dem Abwasser davon, doch wie sehr vermissen wir die Dinge, die niemals wiederkehren. Der Schnee von einst, die Rosen, die heute Morgen aufgeblüht sind und am Abend verblüht sein werden, und wenn erneut die Sonne darauf scheint, werfen sie die Blütenblätter ab, zurück bleiben hässliche trockene Kugeln, kleine Totenköpfe, die zwischen den Fingern knistern, wenn man draufdrückt, die Infanten von Olba, die Damen aus der Ukraine. Wo sind all diese Leute hin, die hastig vor unseren Augen vorbeizogen, wo gingen sie hin, wo sind sie angekommen. Wasser, das der Spülstein schluckt, ein Labyrinth von Abwasserohren, Kloaken, Filtern und Klärbecken, Rohre, die ins ewige Meer führen (Ploetz, versión ebook, s.p.).

[...] è arrivata l'ora dei piagnistei e dei denti che scricchiolano, dei pentimenti amari: dove sono finiti gli euro di una volta? Cosa ne è 
stato di quei bigliettoni violacei? Sono volati via come le foglie morte in autunno quando tira il vento, e sono finiti a marcire nel fango: sono cascati sui tavoli da gioco, tra le chele degli astici e delle granseole che facevamo scrocchiare con le pinze per crostacei [...], sui letti dei bordelli; sulle cassette degli sciacquoni nei bagni, con tutto quello spolverio di polvere bianca [...]: quei fruscianti bigliettoni violacei da cinquecento euro, dove saranno mai finiti? Tutti li cercano e nessuno li trova, li cerchiamo noi imprenditori, li cercano i funzionari delle tasse, qui non ci sono, là neppure. [...] Tutto è finito negli scarichi, nei lavandini, nei cessi, nelle vagine ancora in fiore o incallite a furia di sfregarle. La stessa vita, la nostra, non credere che finisca da un'altra parte, il mondo intero scorre ormai nelle fognature, e quanto ci mancano tutte queste cose che non torneranno più. La neve di un tempo, le rose sbocciate stamattina e già appassite nel pomeriggio, e quando il sole tornerà a risplendere tutti i petali saranno caduti, resterà un grumo secco, piccoli teschi che scricchiolano tra le dita se li tocchi, i ragazzini di Olba, le signore dell'Ucraina. Dove sarà finita tutta quella gente che passava frettolosamente davanti ai nostri occhi, dove andava, cosa faceva. Acqua inghiottita dallo scarico, un labirinto di tubature, cloache, filtri depuratori, canali di scolo che finiscono in mare (Cacucci, pp. 371-372).

[...] l'heure est aux pleurs et aux grincements de dents, aux regrets : où sont les euros d'antan? Qu'a-t-on fait des beaux billets violets? Ils sont tombés d'un coup, comme les feuilles mortes en automne par un jour de grand vent, et ils ont pourri dans la boue : ils sont tombés sur les tables de jeu, entre les pinces des homards et des tourteaux que nous faisions craquer, nous-mêmes armés d'une pince à décortiquer les fruits de mer [...], sur les plumards des bordels ; sur les chasses d'eau, où on a aligné de la poudre blanche en pagaille [...] : quid des beaux billets violets de cinq cents, où sont-ils ? Où sont-ils allés se nicher? Tout le monde les cherche et personne ne les trouve, nous les entrepreneurs, on les cherche, les fonctionnaires des finances les cherchent, rien par-ici, rien par-là. [...] Tout est parti par les lavabos, par les éviers, dans les toilettes, par le trou de cons à peine en fleur et déjà calleux à force de frottements. La vie même, la nôtre, ne t'imagine pas non plus qu'elle s'en va par un autre endroit, le monde entier se déverse dans le tout-à-l'égout, mais combien nous les regrettons, toutes ces choses qui ne reviendront plus. Les neiges d'antan, les 
roses qui se sont ouvertes ce matin seront fanées ce soir et, quand le soleil les caressera de nouveau, elles auront perdu leurs pétales, laides boules desséchées, petites têtes de mort qui craquent entre les doigts quand tu les écrases, infants d'Olba, dames d'Ukraine. Où sont tous ces gens qui passaient vite devant nos yeux, où allaient-ils, où ont-ils fini ? De l'eau qu'avale l'évier, labyrinthe de canalisations, d'égouts, de filtres et de bassins de décantation, tuyaux qui finissent tous dans la mer (Laroutis, versión ebook, láminas 6258-6281)

[...] it's a time of wailing and gnashing of teeth, a time of repentance: where are the euros of yesteryear? What became of those lovely purple notes? They fell as fast as dead leaves on a windy autumn day and rotted in the mud: they fell on the casino tables, onto the claws of the lobsters and crabs we used to crack open with a nutcracker [...], they fell on the rickety beds in brothels; on the lines of white powder left on toilet cisterns [...]: those lovely purple 500 euro notes, ubi sunt? Where are they? Everyone's looking for them and no one can find them, those of us who are entrepreneurs are looking for them and so are the taxmen, but they're nowhere to be found. [...]. It all went down the drain, down the toilet, into the holes of cunts just coming into bloom and already grown calloused from all that friction. Do you really think it's any different with life itself, our life? The whole world is being washed down the drain, but how we miss all those things that will never come back. The snows of yesteryear, the rose that opened this morning and will have faded by evening, and whose petals will fall when the sun shines on it tomorrow, leaving only an ugly dry ball, a miniature skeleton that crunches between your fingers when you squeeze, the princes of Olba, the ladies of Ukraine. Where did they go, all those people who passed so swiftly before our eyes, where were they going, where did they end up? Water swallowed by the drain, by the labyrinth of pipes, sewers, filters and water treatment plants, pipes that flow out into the sea (Jull Costa, 2016, pp. 471-473).

Consideremos ahora cómo los traductores han abordado las principales referencias intertextuales del texto de partida:

Biblia: la expresión "llanto y crujir de dientes" (que es traducción usual del "fletus et stridor dentium" de $M t 8,12)$ es traducida por Ploetz, Laroutis 
y Jull Costa con sintagmas inmediatamente reconocibles en las culturas de llegada (en el caso del alemán, se trata de la versión de Lutero, y por otra parte "gnashing of teeth" ya se encuentra en la Biblia del rey Jacobo). Cacucci, en cambio, rechaza el esperable "pianto e stridore di denti” por “piagnistei [que en italiano significa más bien 'gimoteos' o 'lamentos'] e [...] denti che scricchiolano", que alteran la transparencia del original. A la luz de la ampliación de "arrepentimientos" en "pentimenti amari" (que en efecto mejora la prosodia del período), puede conjeturarse que de entrada el traductor quería evitar una cacofonía por proximidad ("stridore di denti"/"pentimenti"); el mismo resultado hubiera podido conseguirse, sin embargo, con "denti che stridono".

Villon: "Las nieves de antaño" viene del conocido estribillo de la Ballade des dames du temps jadis, "Mais ou sont les neiges d'anten?" ${ }^{10}$. Como es natural, Laroutis reinstaura el sintagma francés, mientras que con "The snows of yesteryear" Jull Costa incorpora con acierto la famosa traducción de Dante Gabriel Rossetti, The Ballad of Dead Ladies"1, con el "yester-year", acuñado precisamente para verter el tan sugestivo "anten" de Villon ${ }^{12}$. En este sentido, parecen menos satisfactorias las soluciones de Cacucci ("La neve di un tempo" en lugar de "le nevi dell'altr'anno" o "le nevi dell'anno passato"13) y Ploetz ("Der Schnee von einst", que desecha el "Wo ist der Schnee vom vergangenen Jahr?" utilizado por Brecht en la Lied de Nanna).

10 Cito por François Villon, Opere, edición de Emma Stojkovic Mazzariol, trads. Attilio Carminati y Emma Stojkovic Mazzariol, nueva edición revisada y aumentada, Milano, Mondadori, 2000, p. 62.

11 Dante Gabriel Rossetti, Poems and Translations. 1850-1870, London, Oxford University Press, 1968, pp. 101-102.

12 Observaba Stojkovic Mazzariol: "Le donne celebri del passato, dove sono? Ma sarebbe come chiedere dove sono le nevi dell'anno appena trascorso! Una domanda assurda, vuol dirci il poeta, perché le une come le altre non sono in nessun luogo: sono scomparse in un lampo, dissolte, riassorbite nell'eterna vicenda del cosmo. Proprio la scelta di una temporalità recente (anten) e di un elemento naturale labile come la neve, accentua il concetto della fragilità e brevità della vita umana" (François Villon, Opere, p. 430).

13 Son las traducciones, respectivamente, de Carminati y Stojkovic Mazzariol (François Villon, Opere, p. 63) y de Roberto Mussapi (François Villon, Ballate del tempo che se ne andì, Milano, il Saggiatore, 2008, p. 35). 
Ahora bien, el lector más atento ya había topado con una alusión villoniana unas cuatrocientas páginas atrás, cuando Esteban habla del bar Castañer, donde ya no se puede fumar: "la protectora gasa del humo del tabaco, que hoy, como las damas de antańo, se ha desvanecido" ${ }^{14}$, donde se alude evidentemente a las dames du temps jadis de la balada de Villon. Mientras que Ploetz y Laroutis consiguen señalar la referencia ("die Damen von einst", "les dames du temps jadis"), Cacucci propone "le donne di una volta" ('las mujeres de hace tiempo') ${ }^{15}$, con lo cual el guiño literario se hace prácticamente invisible para el lector italiano. El caso más interesante se da sin embargo en la traducción de Jull Costa, quien cambia el original por otro "the snows of yesteryear", manteniendo así tanto el motivo del ubi sunt como la intertextualidad villoniana, pero a costa de sacrificar la alusión a las mujeres, lo que parece poco adecuado para la coherencia interna de un texto tan imbuido de ańoranzas y rencores sentimentales y/o carnales.

Manrique: he dejado para el final la cuestión de la presencia de las Coplas porque creo que plantea problemas de estrategia traductora algo más complejos que los casos anteriores. El primero deriva naturalmente de la competencia cultural básica que es posible asumir pensando en un genérico lector español, frente a los hipotéticos conocimientos enciclopédicos de un homólogo francés, italiano, alemán, británico o americano ${ }^{16}$. Como apuntaba Senabre, es posible incluso que en un futuro próximo las alusiones al poema de Manrique se tornen incomprensibles para el gran público nacional, pero de momento, aunque los versos de las Coplas hayan dejado de resonar "en todas las memorias españolas" ${ }^{17}$, es plausible que el lector medio de Chirbes sepa detectar con facilidad el hipotexto

14 Rafael Chirbes, En la orilla, p. 60.

15 Carminati y Stojkovic Mazzariol traducen "dame d'un tempo", Mussapi "donne del tempo passato".

16 Es un problema que, por ejemplo, se planteó legítimamente Jull Costa al traducir "polvo seremos, pero yo seré polvo - como el del poeta- enamorado" (En la orilla, p. 364) como "I will be dust, but, as Quevedo says, I will be dust in love" (p. 400).

17 Así se expresaba Pierre le Gentil, refiriéndose al íncipit del poema, en La poésie lyrique espagnole et portugaise à la fin du Moyen Âge de 1949 (reproducido parcialmente en Jorge Manrique, Poesía, ed. Vicente Beltrán, Barcelona, Crítica, 1993, pp. IX-XXVIII [p. XXVII]). 
manriqueño, por lo menos gracias a recuerdos escolares. Por si el reconocimiento no fuera inmediato, Chirbes desperdigó arcaísmos lingüísticos (“qqué se hizo...?”, la variante "que van a dar a la mar") o sintagmas inusuales ("los infantes de Olba, las damas de Ucrania") que facilitan la identificación de un pastiche, además de subrayar socarronamente con el “ubi?” el tópico moral de la secuencia.

Pues bien, es posible constatar empíricamente, a partir de las traducciones de nuestro corpus, cómo estos elementos, bien perceptibles en el texto original, corren el riesgo de hacerse opacos o desaparecer por completo cuando se vierten a otro idioma.

Los defectos más importantes se notan en la traducción de Cacucci, que, además de omitir sin motivos aparentes el "ubi?", tergiversa totalmente el significado de "los infantes de Olba, las damas de Ucrania", que pasa a un poco comprensible "i ragazzini di Olba, le signore dell'Ucraina" ('los chiquillos de Olba, las señoras de Ucrania'). Asimismo, "en qué pararon" (que parece remedar el “¿cuál se para?” de las Coplas, v. 102) ${ }^{18}$ se traduce con un incorrecto "cosa facevano"19. Más allá de los errores de traducción, sin embargo, habría que preguntarse si, a falta de un comentario paratextual, una traducción fundamentalmente modernizadora, que allana las irregularidades diacrónicas de la lengua original, no acaba por ocultar el juego de referencias. Me refiero en particular a la frase que termina en "que van a dar a la mar", que en las traducciones italiana, inglesa y francesa queda completamente opaca, en el caso de no conocer el hipotexto manriqueńo. Creo que es este el motivo por el que Ploetz, con un giro acertado, amplió “a la mar” en "ins ewige Meer” ('en el mar eterno').

Una de las soluciones recomendadas por Umberto Eco para mantener este tipo de guiños intertextuales consiste en recurrir a traducciones preexistentes del texto aludido en la lengua meta ${ }^{20}$, operación que naturalmente no siempre resulta practicable o fructífera. En el caso del in-

18 Cito por la edición de Vicente Beltrán indicada en la nota anterior.

19 Añádase, al margen de las observaciones sobre Manrique, que la disyuntiva inoportunamente introducida en la frase "nelle vagine ancora in fiore $o$ incallite a furia di sfregarle" (por "los cońos apenas en flor y ya encallecidos de tanto frotar": ¿por qué el eufemismo, además?) malogra el paralelo con las barrocas "rosas que se han abierto esta mańana y se marchitarán a la tarde".

20 Cf. Umberto Eco, Dire quasi la stessa cosa [2003], Milano, Bompiani, 2014, p. 217. 
glés, por ejemplo, la célebre versión decimonónica de Henry Wadsworth Longfellow traduce "Nuestras vidas son los ríos / que van a dar en el mar / que es el morir" como "Our lives are rivers, gliding free / To that unfathomed, boundless sea, / The silent grave!"21, versos que, a pesar de su prestigio cultural, sería difícil adaptar al pastiche de Chirbes. Pesa también, para el traductor, la responsabilidad de proponer una versión deliberadamente distanciadora, que incluso podría pasar por torpe o incorrecta: en efecto, volviendo a la traducción italiana, el ya mencionado "finiscono in mare" podría sustituirse por "mettono foce nel mare" o "vanno a dare nel mare" 22 , a costa de unas abstractas 'naturalidad' y 'fluidez' del texto.

21 The Poems of Henry Wadsworth Longfellow, New York, Harper and Brothers, 1846, p. 17.

22 La primera solución es de Mario Pinna (Jorge Manrique, Poesie, Firenze, Vallecchi, 1962, p. 61), la segunda la comparten Luciano Allamprese (Jorge Manrique, Stanze per la morte del padre, Torino, Einaudi, 1991, p. 3) y Giovanni Caravaggi (Jorge Manrique, Elegia alla morte del padre, Venezia, Marsilio, 1991, p. 39). 